\title{
Prognostic implications of stromal hyaluronic acid protein expression in resected oropharyngeal and oral cavity cancers
}

\author{
Der Sheng Sun ${ }^{1, *}$, Hye Sung Won ${ }^{1, *}$, Soon Auck Hong ${ }^{2}$, Ji Hyung Hong ${ }^{1}$, Heejoon Jo ${ }^{3}$, Heejin Lee ${ }^{4}$, \\ Okran Kim ${ }^{4}$, Myung Ah Lee ${ }^{1,4}$, and Yoon Ho $\mathrm{Ko}^{1,4}$
}

\begin{abstract}
${ }^{1}$ Division of Oncology, Department of Internal Medicine, College of Medicine, The Catholic University of Korea, Seoul; ${ }^{2}$ Department of Pathology, Soonchunhyang University Cheonan Hospital, Cheonan, Korea; ${ }^{3}$ Department of Biostatistics, University of North Carolina at Chapel Hill, Chapel Hill, NC, USA; ${ }^{4}$ Cancer Research Institute, College of Medicine, The Catholic University of Korea, Seoul, Korea
\end{abstract}

Received: June 9, 2018 Revised : August 10, 2018 Accepted: August 14, 2018

\section{Correspondence to \\ Yoon Ho Ko, M.D.}

Division of Oncology,

Department of Internal

Medicine, Uijeongbu St. Mary's

Hospital, College of Medicine,

The Catholic University of Korea,

271 Cheonbo-ro, Uijeongbu 11765,

Korea

Tel: $+82-31-820-3985$

Fax: +82-31-847-2719

E-mail: koyoonho@catholic.ac.kr

*These authors contributed equally to this work.
Background/Aims: Hyaluronic acid (HA) regulates cell adhesion, migration and proliferation in various cancers. The clinical implications of HA in resected head and neck squamous cell carcinoma have not been elucidated. We investigated the clinical significance and prognostic value of the expression of tumoral and stromal HA and its related proteins in oropharyngeal and oral cavity cancer.

Methods: Resected tissues from oropharyngeal or oral cavity cancer patients undergoing surgery were analysed in tissue microarrays divided into stroma and cancer panels. The expression levels of HA, HA synthases and hyaluronidases were also assessed by immunohistochemistry.

Results: A total of 160 resected oropharyngeal or oral cavity cancer tissues were analysed. Stromal HA expression was observed more frequently in human papilloma virus (HPV)-negative tumors, but other clinicopathological characteristics did not differ. In patients with HPV-negative oral cavity cancers, high stromal HA expression was associated with significantly shorter recurrence-free survival and overall survival compared with low stromal HA expression. The expression of HA in both tumors and stroma was significantly correlated with poorer outcomes than other combinations in patients with HPV-negative oral cavity cancers. However, these prognostic roles of HA were not observed in patients with HPV-negative oropharyngeal cancers. In the HPV-stratified multivariate analysis, high stromal HA expression remained an independent indicator of poor prognosis in terms of recurrence-free survival.

Conclusions: High stromal HA and expression of HA in both tumors and stroma were correlated with poor prognosis in HPV-negative oral cavity cancer, but not in HPV-negative oropharyngeal cancers.

Keywords: Hyaluronic acid; Prognosis; Stroma; Human papillomavirus; Head and neck neoplasms

\section{INTRODUCTION}

Head and neck squamous cell carcinoma (HNSCC) is the most common malignant neoplasm of the head and neck [1]. A multimodal treatment strategy has been widely considered the standard treatment for locally advanced HNSCC. With recent advancements in our understanding of the molecular biology of HNSCC, agents 
targeting specific molecular markers, such as epidermal growth factor receptor, have also been widely used with cytotoxic chemotherapy or radiotherapy to improve patient survival in the setting of locally advanced and recurrent HNSCC [2], but the survival of HNSCC patients is still unsatisfactory. The tumor microenvironment associated with the process of HNSCC has been attracting attention in investigations of effective prognostic factors in HNSCC [3].

The tumor microenvironment is composed of cancer-associated fibroblasts, immune cells, and other stromal cells around the cancer cell extracellular matrix (ECM), which includes cell-adhesion molecules and ligands and plays a role in tissue organisation [4]. Hyaluronic acid (HA), composed of alternating N-acetylglucosamine and glucuronic acid units, is one of the major carbohydrates in the ECM. HA-family molecules include HA-synthase, HA receptors, and hyaluronidases (HAases). HA is synthesised by HA synthases (HAS1, HAS2, and $\mathrm{HAS}_{3}$ ) and degraded by HAases (such as HYAL1) $[5,6]$. There are six HAases in the human genome, and HYAL1 and HYAL2 have the highest enzymatic activity. HA participates in tumor cell invasion and metastasis, and regulates cell proliferation, migration, and angiogenesis by interacting with specific cell-surface receptors, including CD44 and the receptor of HA-mediated motility [4].

HA expression is known to be more abundant in stroma than in cancer cells, in various carcinomas [7]. As a prognostic factor, although HA in neoplastic cells predicted poor survival in patients with various cancers [8,9], elevated HA expression in tumor stroma also predicted poor survival in patients with breast cancer or prostate cancer [10]. However, there have been few reports on the prognostic significance of $\mathrm{HA}$ in oropharyngeal squamous cell carcinoma (OPSCC) or oral cavity squamous cell carcinoma (OSCC). In this study, we investigated the clinical significance and prognostic value of the expression of HA and its related molecules in patients with OPSCC and OSCC who underwent surgical resection.

\section{METHODS}

\section{Patients}

We retrospectively analysed the clinical and patholog- ical data of patients diagnosed with OSCC and OPSCC who underwent surgery at Seoul St. Mary's Hospital and Uijeongbu St. Mary's Hospital of the Catholic Medical Center (Seoul, Korea) between 1994 and 2012. The inclusion criteria were a pathological diagnosis of HNSCC of the oropharynx or oral cavity, curative surgical resection, available tissue for HA expression analysis and available clinical pathological data, including patient demographics, smoking history, site of primary tumor, pathological tumor stage and patient outcomes, such as the dates of death, last follow-up and relapse. Patients who underwent pre-operative radiation or chemotherapy were ineligible. All patients underwent surgery and received appropriate adjuvant chemotherapy and/or radiotherapy where necessary. Pathological staging was determined according to the 7 th edition of the American Joint Committee on Cancer staging criteria. Informed consent was waived because of the retrospective study using tissues before 2013. The study was approved by the Institutional Review Board of the Catholic Medical Center (Ref. No. KC15SISO4O4).

\section{Tissue microarrays}

For tissue microarray (TMA) analyses, two representative tumor areas were selected on haematoxylin and eosin (H\&E)-stained slides. Duplicate cylindrical cores with 2-mm diameters were obtained from morphologically representative tissues of each tumor paraffin block using a manual tissue arrayer (SuperBioChips Laboratories, Seoul, Korea) and assembled in a TMA format. A section from each TMA block was stained with H\&E and subjected to histological examination. Sections $4-\mu \mathrm{m}$ in thickness were used for immunohistochemical staining.

\section{Immunohistochemical staining}

Immunohistochemistry was performed using the Ventana Benchmark XT platform (Ventana Medical System, Tucson, AZ, USA) and the Ultraview DAB detection system (Ventana). Primary antibodies for HA (1:300, Abcam, Cambridge, UK), HYALı (1:200, Abcam), HAS2 (1:100, Biorbyt, Ltd., Cambridge, UK), and p16 (JC8, 1:200, Santa Cruz Biotechnology, Santa Cruz, CA, USA) were used. Primary tumoral and stromal molecule expression was analysed based on intensity and proportion. The staining intensity was scored semi-quantitatively as 0 , no staining; 1, weakly positive; 2 , moderately positive; or 
3, strongly positive. The proportion was evaluated as positive tumor cell percentage. The histological score (H score; o to 300) was calculated by multiplying the intensity and proportion of expression. To determine the optimal cut-off values for survival prediction, a maximally selected rank statistic test was performed using the R Maxstat Package version 3.3.1 (R Foundation for Statistical Computing, Vienna, Austria). As a result, high expression was determined as follows: $\mathrm{H}$ score $>10$ for tumoral HA expression, $>40$ for tumoral HYALı expression, and $>30$ for tumoral HAS2 expression. In tumor stromal expression, the following values were considered positive: $\mathrm{H}$ score $>\mathrm{O}$ for stromal HA expression, and $>10$ for stromal HYALı expression. To assess human papilloma virus (HPV) status, p16 immunostaining was evaluated and strong nuclear and cytoplasmic staining in $\geq 75 \%$ of the tumor cells indicated positive expression. The results were analysed by a board-certified pathologist (S.A.H.) who was blinded to the clinical data.

\section{$H A S_{1}$ and $H A S_{2}$ gene expression according to mutational status of various genes in The Cancer Genome Atlas HNSCC database}

To identify associations between the HAS1 or HAS2 genes and various mutations and clinical features in HNSCC, the open access data tier generated by The Cancer Genome Atlas (TCGA) research network was used. mRNA expression data, mutation annotation files and clinical data were downloaded from the TCGA data portal (https://tcgadata.nci.nih.gov/tcga/). All mRNA expression data were $\log 2$-transformed. The epithelial-mesenchymal transition (EMT) score for each tumor was defined as the sum of the expression of well-known mesenchymal marker genes minus the expression of known epithelial genes, which are based on a previously reported expression signature of 76 genes [11].

\section{Statistical analysis}

Clinicopathological characteristics are presented as medians (range) for continuous variables or numbers (percentage) for categorical variables. Comparisons between two groups were performed using Student's unpaired $t$ test or the chi-square test. Recurrence-free survival (RFS) was measured from the date of surgery to the date of recurrence or censored at the last follow-up date. Overall survival (OS) was measured from the date of surgery until death resulting from any cause or the last censored date during follow-up. RFS and OS were calculated using the Kaplan-Meier method, and differences in survival between groups were compared by the logrank test. Cox proportional hazards regression models were used to investigate the significance of prognostic factors. Variables with $p<0.25$ in the univariate model and/or variables associated with prognosis in HNSCC were included in the multivariate model. A backward elimination process was used to develop the final multivariate model, and adjusted hazard ratios (HRs) with 95\% confidence intervals (CIs) were calculated. A p < 0.05 was considered statistically significant. All statistical analyses were performed using the $\mathrm{R}$ statistical software package version 3.2.3 (R Foundation for Statistical Computing).

\section{RESULTS}

\section{Baseline characteristics}

A total of 160 tumor specimens were included and analysed in this study. The baseline characteristics of the 160 patients are summarised in Table 1 . The median age was 56 years (range, 26 to 90), and $78.8 \%$ were male. A total of 101 patients $(64.3 \%)$ were ever-smokers. Pathological analyses revealed that 130 patients $(81.8 \%)$ were classified as stage $\mathrm{T} 1$ or $\mathrm{T} 2,80(50.0 \%)$ as stage $\mathrm{N} 2$ and $105(65.6 \%)$ as pathological stage III or IV. Based on p16 immunostaining results, 33 patients $(21.9 \%)$ were considered HPV-positive. For primary treatment, 84 patients (52.5\%) received surgery alone, and 76 patients $(47.5 \%)$ received adjuvant treatment after surgery. Standard indications for adjuvant treatment included multiple lymph node involvement, a close or positive resection margin or the presence of extracapsular spread. Adjuvant treatments included radiotherapy alone $(\mathrm{n}=4 \mathrm{O})$, platinum-based chemotherapy $(n=4)$, or concurrent chemoradiotherapy with cisplatin and/or fluorouracil $(n=32)$.

\section{HA family protein expression and relationships with clinicopathological findings}

Cytoplasmic expression of HA, HYAL1 and HAS2 was observed. HA and HYAL1 expression in the tumor stroma was only found when tumor cells were also positive (Fig. 1). The expression levels of the HA family mole- 
Table 1. Clinicopathological characteristics of patients

\begin{tabular}{|c|c|}
\hline Variable & Value \\
\hline No. of patients & $160(100)$ \\
\hline Age, yr, median (range) & $56(26-90)$ \\
\hline \multicolumn{2}{|l|}{ Sex } \\
\hline Male & $126(78.8)$ \\
\hline Female & $34(21.2)$ \\
\hline \multicolumn{2}{|l|}{ Smoking status } \\
\hline Non-smoker & $56(35.7)$ \\
\hline Current/Ex-smoker & $101(64 \cdot 3)$ \\
\hline \multicolumn{2}{|l|}{ Primary site } \\
\hline Oral cavity & $84(52.5)$ \\
\hline Oropharynx & $76(47.5)$ \\
\hline \multicolumn{2}{|l|}{ T stage } \\
\hline $\mathrm{T} 1-2$ & $130(81.8)$ \\
\hline $\mathrm{T}_{3}-4$ & $29(18.2)$ \\
\hline \multicolumn{2}{|l|}{$\mathrm{N}$ stage } \\
\hline No-1 & $80(50.0)$ \\
\hline $\mathrm{N} 2$ & $80(50.0)$ \\
\hline \multicolumn{2}{|l|}{ Stage } \\
\hline I & $33(20.6)$ \\
\hline II & $22(13.8)$ \\
\hline III & $25(15.6)$ \\
\hline IV & $80(50.0)$ \\
\hline \multicolumn{2}{|l|}{ Differentiation } \\
\hline Well & $57(35.8)$ \\
\hline Moderate & $86(54.1)$ \\
\hline Poor & $16(10.1)$ \\
\hline \multicolumn{2}{|l|}{ Lymphatic invasion } \\
\hline Yes & $86(58.1)$ \\
\hline No & $62(41.9)$ \\
\hline \multicolumn{2}{|l|}{ Venous invasion } \\
\hline Yes & $15(10.1)$ \\
\hline No & $133(89.9)$ \\
\hline \multicolumn{2}{|l|}{ Perineural invasion } \\
\hline Yes & $24(16.2)$ \\
\hline No & $124(83.8)$ \\
\hline \multicolumn{2}{|l|}{ HPV status } \\
\hline Positive & $33(21.9)$ \\
\hline Negative & $118(78.1)$ \\
\hline
\end{tabular}

Values are presented as number (\%).

HPV, human papilloma virus. cules were dichotomised based on optimal cut-off values for clinical outcomes. High tumor cell expression of $\mathrm{HA}$ (H score $>10$ ) was found in $47.5 \%$ of samples $(n=76)$, high expression of HYAL1 ( $\mathrm{H}$ score $>40)$ in $38.7 \%(n=62)$ and high HAS2 (H score $>30)$ in 10.6\% $(n=17)$. Positive stromal expression of $\mathrm{HA}$ (any expression in the tumor stroma) was found in $19.4 \%$ of patients $(n=31)$, and positive HYALı expression (H score > 10) was found in 10.0\% $(\mathrm{n}=16)$. No HAS2 expression was observed in stromal tissues. Positive HA expression in tumor stroma was significantly correlated with tumoral HA expression ( $p$ $<0.001$ ) and stromal HYAL1 expression $(p=0.016)$ (Table 2). The relationship between stromal HA expression and clinicopathological findings was explored (Table 3). In all cases, stromal HA expression was not significantly correlated with HPV infection, but displayed a trend, and no significant differences were observed between stromal HA expression and age, gender, primary tumor site, histological differentiation, or lymph node metastasis.

\section{Clinicopathological factors associated with prognosis stratified by HPV status}

During the median follow-up of 59.6 months (range, 2.2 to 241.3$), 56$ patients (35.0\%) developed tumor recurrence, and 86 (53.7\%) died from any cause. Recurrence patterns included local recurrence in 40 cases (19.6\%), distant recurrence in 17 cases (8.3\%) and both local and distant recurrence in six cases $(2.9 \%)$. The overall 5 -year RFS rate for patients was $28.2 \%$. The median RFS time was 14.3 months (range, 11.6 to 17.0) for all patients. HPV-related HNSCC has a molecular, epidemiological and clinical profile that is distinct from classical tobacco and/or alcohol-associated HNSCCs [12], and HPV infection is a well-known powerful predictive and prognostic indicator in HNSCC, especially in oropharyngeal cancer [12]. Therefore, to eliminate the influence of HPV infection on prognosis, we performed HPV-stratified analyses to assess the independent prognostic value of the HA family. Based on the univariate analyses, predictors of RFS included advanced pT stage $(p=0.001)$, advanced pN stage $(p=0.001)$, lymphatic invasion status $(p=0.017)$, smoking status $(p=0.041)$, and poor differentiation $(p=$ 0.048) (Table 4). In the multivariate analysis of RFS, advanced $\mathrm{pT}$ stage $(\mathrm{HR}, 2.591 ; p=0.011)$ and advanced $\mathrm{pN}$ stage $(H R, 2.232 ; p=0.048)$ remained significant prog- 

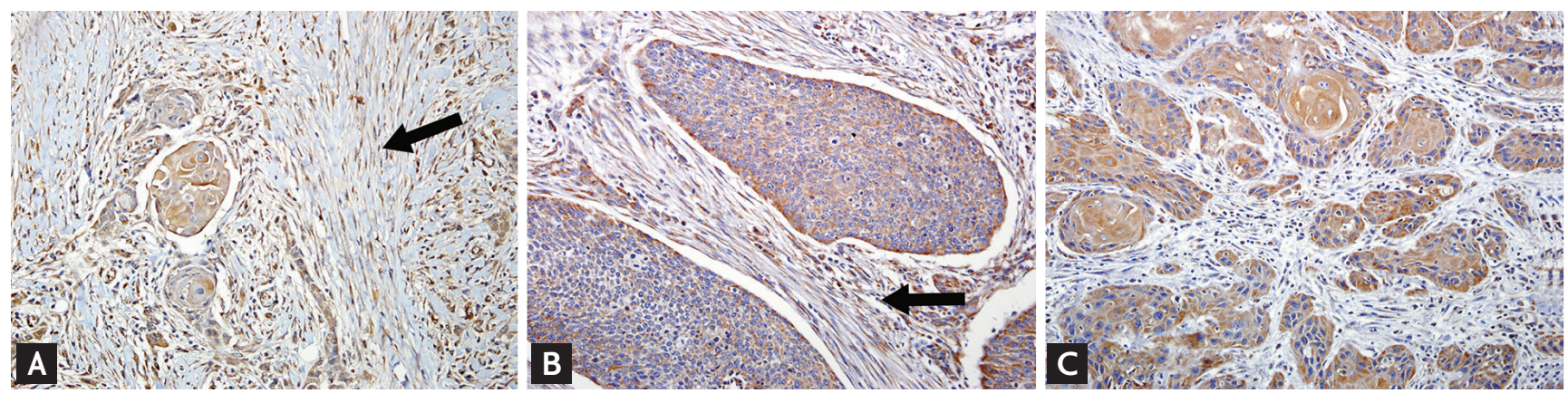

Figure 1. Immunohistochemical staining for hyaluronic acid (HA) (A) and hyaluronidase 1 (HYAL1) (B) shows co-expression in squamous cell carcinoma and tumor stroma (arrow), whereas HA synthase 2 (HAS2) (C) expression is only present in tumor cells.

Table 2. Correlations of hyaluronic acid family molecules in tumors and stroma

\begin{tabular}{|c|c|c|c|c|}
\hline \multirow{2}{*}{ Variable } & \multirow{2}{*}{ Incidence } & \multicolumn{2}{|c|}{ HA, stroma } & \multirow{2}{*}{$p$ value } \\
\hline & & Negative & Positive & \\
\hline Total patients & $160(100)$ & $129(80.6)$ & $31(19.4)$ & \\
\hline HA, tumor & & & & $<0.001$ \\
\hline Low & $84(52.5)$ & $79(61.2)$ & $5(16.1)$ & \\
\hline High & $76(47.5)$ & $50(38.8)$ & $26(83.9)$ & \\
\hline HYALı, tumor & & & & 0.538 \\
\hline Low & $98(61.3)$ & $77(59.7)$ & $21(67 \cdot 7)$ & \\
\hline High & $62(38.7)$ & $52(40.3)$ & $10(32.3)$ & \\
\hline HYAL1, stroma & & & & 0.016 \\
\hline Low & $144(90.0)$ & $120(93.0)$ & $24(77.4)$ & \\
\hline High & $16(10.0)$ & $9(7.0)$ & $7(22.6)$ & \\
\hline HAS2, tumor & & & & 0.745 \\
\hline Low & $143(89.4)$ & $116(89.9)$ & $27(87.1)$ & \\
\hline High & $17(10.6)$ & $13(10.1)$ & $4(12.9)$ & \\
\hline
\end{tabular}

Values are presented as number (\%).

HA, hyaluronic acid; HYAL1, hyaluronidase 1; HAS2, HA synthases 2.

nostic factors. Age, advanced T or N stage, lymphatic invasion and venous invasion status were associated with poor OS (Table 5). Of these factors, age (HR, 1.038; $p=$ $0.004)$, advanced $\mathrm{pT}$ stage (HR, 2.425; $p=0.002)$, and advanced $\mathrm{pN}$ stage $(\mathrm{HR}, 1.833 ; p=0.023)$ were identified as independent prognostic factors of poor OS in multivariate analysis.

\section{Prognostic role of HA family molecule expression stratified by HPV status}

In patients with HPV-positive oral cavity or oropharyngeal cancers, the expression of HA family molecules in tumors and stroma showed no significant correlations with RFS or OS (oral cavity cancer: RFS, $p=0.414$; OS, $p=0.886$; oropharyngeal cancer: RFS, $p=0.581 ;$ OS, $p=$ 0.519). Regarding clinical outcomes of patients with HPV-negative oral cavity cancers, high stromal HA expression resulted in significantly shorter RFS $(p<0.001$, Fig. $2 \mathrm{~A}$ ) and $\mathrm{OS}(p=0.040$, Fig. $2 \mathrm{C})$ compared with low stromal HA expression as determined by log-rank test. In patients with HPV-negative oropharyngeal cancers; however, stromal HA expression showed no significant correlation with RFS ( $p=0.453$, Fig. $2 \mathrm{~B})$ or OS $(p=0.230$, Fig. 2D). A significant and strong relationship between 
Table 3. Characteristics of patients by stromal HA expression

\begin{tabular}{lccc}
\hline \multirow{2}{*}{ Variable } & \multicolumn{2}{c}{ HA, stroma } & \multirow{2}{*}{ p value } \\
\cline { 2 - 3 } & Negative & Positive & \\
\hline Primary site & & & \\
Oral cavity & $68(52.7)$ & $16(51.6)$ & \\
Oropharynx & $61(47.3)$ & $15(48.4)$ & \\
T stage & & & 0.451 \\
T1-2 & $106(82.8)$ & $24(77.4)$ & \\
T3-4 & $22(17.2)$ & $7(22.6)$ & \\
N stage & & & 0.690 \\
No-1 & $66(51.2)$ & $14(45.2)$ & \\
N2 & $63(48.8)$ & $17(54.8)$ & \\
Stage & & & \\
I & $28(21.7)$ & $5(16.1)$ & \\
II & $17(13.2)$ & $5(16.1)$ & \\
\hline III & $21(16.3)$ & $4(12.9)$ & \\
IV & $63(48.8)$ & $17(54.8)$ & \\
Differentiation & & & \\
Well & $47(36.4)$ & $10(33.3)$ & \\
\hline Moderate & $69(53.5)$ & $17(56.7)$ & \\
Poor & $13(10.1)$ & $3(10.0)$ & \\
HPV status & & & \\
\hline Positive & $29(24.2)$ & $4(12.9)$ & \\
Negative & $91(75.8)$ & $27(87.1)$ & \\
\hline
\end{tabular}

Values are presented as number (\%).

HA, hyaluronic acid; HPV, human papilloma virus.

tumor and stromal HA expression was observed; therefore, we analysed the expression of HA in tumors and stroma. In patients with HPV-negative oral cavity cancers, the expression of HA in both tumors and stroma was significantly correlated with poorer outcomes than other combinations (RFS, $p=0.069$, Fig. 3A; OS, $p=$ 0.015 , Fig. ${ }_{3} \mathrm{C}$ ), but this was not the case in patients with HPV-negative oropharyngeal cancers (RFS, $p=0.742$, Fig. ${ }_{3} \mathrm{~B}$; OS, $p=0.671$, Fig. $\left.3 \mathrm{D}\right)$. In the HPV-stratified multivariate analysis, high stromal HA expression remained an independent indicator of poor prognosis in terms of RFS (HR, 2.712; 95\% CI, 1.323 to 5.56; $p=0.006$ ) (Table 4), but not OS (Table 5).

HAS1 and HAS2 gene expression in the TCGA database The gene expression levels of HAS1 and HAS2 were ana- lysed in HNSCC samples $(\mathrm{n}=279)$ from the TCGA database. We compared HAS1 or HAS2 gene expression with primary site, smoking status and HPV status. HAS1 and HAS2 mRNA upregulation was enriched in oral cavity and HPV-negative cancers (oral cavity: $p<0.001$ and $p<$ 0.001 , respectively, Fig. 4A; HPV status: $p=0.004$ and $p$ $=0.005$, respectively, Fig. $4 \mathrm{~B}$ ). HAS1 and HAS2 gene expression were significantly correlated with EMT score (Fig. 4C).

\section{DISCUSSION}

In this study, we investigated the clinical significance of HA-family molecules in patients with oropharyngeal or oral cavity cancer receiving curative surgical resection. Although there were no significant correlations between HA expression and clinicopathological findings, we found that high stromal HA expression was an independent poor prognostic factor in terms of RFS. According to HPV-stratified analysis, patients with high stromal HA expression in HPV-negative oral cavity cancer showed significantly shorter RFS and OS. However, HYAL1 and HAS2 expression showed no significant associations with RFS or OS. These observations suggested that the prognostic impact of stromal HA expression is much stronger in HPV-negative tumors than in HPV-positive tumors.

The tumor microenvironment is composed of ECM and numerous stromal cells. The importance of the tumor microenvironment in cancer initiation and progression is well established. Changes in the ECM occur during cancer development, enabling tumor cells to disseminate [13]. The glycosaminoglycan HA is one of the main components of the ECM. HA levels are maintained through a balance of synthesis by HAS and degradation by the enzyme HYAL1. The roles of HA-family molecules in cancer have been widely investigated. In cancer patients, HA concentrations are usually higher in malignant tumors than in benign and normal tissues. Several studies have reported a correlation between HA concentration in tumors or stroma and tumor aggression [14]. HA synthase and/or HYAL have been suggested to contribute to tumor cell proliferation, motility and invasion by controlling the level of HA and activating intracellular signalling pathways initiated by HA inter- 
Table 4. Prognostic factors for recurrence-free survival in univariate and multivariate analyses with stratification by HPV status

\begin{tabular}{|c|c|c|c|c|}
\hline \multirow{3}{*}{ Variable } & \multicolumn{4}{|c|}{ Recurrence-free survival } \\
\hline & \multicolumn{2}{|c|}{ Univariate } & \multicolumn{2}{|c|}{ Multivariate } \\
\hline & $\operatorname{HR}(95 \% \mathrm{CI})$ & $p$ value & $\operatorname{HR}(95 \% \mathrm{CI})$ & $p$ value \\
\hline Male sex & $0.554(0.260-1.180)$ & 0.126 & $1.438(0.501-4.102)$ & 0.496 \\
\hline $\operatorname{Age}(\geq 56 \mathrm{yr})$ & $1.003(0.976-1.030)$ & 0.852 & & \\
\hline Smoking, yes & $1.917(1.026-3.580)$ & 0.041 & $1.850(0.723-4.731)$ & 0.198 \\
\hline Primary site, oropharynx & $1.103(0.630-1.931)$ & 0.732 & & \\
\hline Differentiation, poor & $1.547(1.004-2.384)$ & 0.048 & $2.032(0.802-5.141)$ & 0.134 \\
\hline T stage, $\geq \mathrm{p}^{\prime} \mathrm{T}_{3}$ & $2.897(1.561-5.378)$ & 0.001 & $2.591(1.236-5.432)$ & 0.011 \\
\hline $\mathrm{N}$ stage, $\geq \mathrm{pN} 2$ & $2.679(1.521-4.717)$ & 0.001 & $2.232(1.004-4.961)$ & 0.048 \\
\hline Lymphatic invasion (+) & $2.092(1.144-3.828)$ & 0.017 & $1.271(0.787-2.661)$ & 0.524 \\
\hline Venous invasion $(+)$ & $1.686(0.712-3.992)$ & 0.235 & & \\
\hline Perineural invasion $(+)$ & $0.967(0.443-2.109)$ & 0.932 & & \\
\hline HA cancer $(+)$ & $1.631(0.948-2.806)$ & 0.077 & $1.592(0.849-2.990)$ & 0.146 \\
\hline HA stroma $(+)$ & $1.999(1.108-3.607)$ & 0.021 & $2.712(1.323-5.560)$ & 0.006 \\
\hline HYALı cancer $(+)$ & $0.942(0.545-1.631)$ & 0.832 & & \\
\hline HYAL1 stroma $(+)$ & $0.393(0.094-1.634)$ & 0.199 & $0.247(0.051-1.200)$ & 0.083 \\
\hline $\mathrm{HAS}_{2}$ cancer $(+)$ & $0.772(0.306-1.948)$ & 0.584 & & \\
\hline
\end{tabular}

HPV, human papilloma virus; HR, hazard ratio; CI, confidence interval; HA, hyaluronic acid; HYALı, hyaluronidase 1 ; HAS2, HA synthases 2 .

Table 5. Prognostic factors for overall survival in univariate and multivariate analyses with stratification by HPV status

\begin{tabular}{|c|c|c|c|c|}
\hline \multirow{3}{*}{ Variable } & \multicolumn{4}{|c|}{ Overall survival } \\
\hline & \multicolumn{2}{|l|}{ Univariate } & \multicolumn{2}{|c|}{ Multivariate } \\
\hline & $\operatorname{HR}(95 \% \mathrm{CI})$ & $p$ value & $\operatorname{HR}(95 \% \mathrm{CI})$ & $p$ value \\
\hline Male sex & $0.608(0.335-1.104)$ & 0.102 & $0.662(0.344-1.28)$ & 0.219 \\
\hline $\operatorname{Age}(\geq 56 \mathrm{yr})$ & $1.041(1.017-1.064)$ & 0.001 & $1.038(1.012-1.07)$ & 0.004 \\
\hline Smoking, yes & $1.344(0.84-2.152)$ & 0.218 & & \\
\hline Primary site, oropharynx & $1.105(0.703-1.738)$ & 0.664 & & \\
\hline Differentiation, poor & $1.067(0.756-1.506)$ & 0.714 & & \\
\hline T stage, $\geq \mathrm{p}^{\prime} \mathrm{T}_{3}$ & $2.902(1.771-4.754)$ & $<0.001$ & $2.425(1.369-4.301)$ & 0.002 \\
\hline $\mathrm{N}$ stage, $\geq \mathrm{pN} 2$ & $2.038(1.303-3.187)$ & 0.002 & $1.833(1.085-3.102)$ & 0.023 \\
\hline Lymphatic invasion (+) & $2.015(1.231-3.301)$ & 0.005 & & \\
\hline Venous invasion $(+)$ & $1.932(1.013-3.685)$ & 0.046 & $1.542(0.783-3.041)$ & 0.210 \\
\hline Perineural invasion $(+)$ & $1.167(0.647-2.105)$ & 0.607 & & \\
\hline HA cancer $(+)$ & $1.380(0.891-2.137)$ & 0.149 & $1.327(0.800-2.200)$ & 0.271 \\
\hline HA stroma $(+)$ & $1.736(1.050-2.871)$ & 0.032 & $1.475(0.806-2.700)$ & 0.207 \\
\hline HYALı cancer $(+)$ & $0.818(0.519-1.289)$ & 0.385 & & \\
\hline HYAL1 stroma (+) & $0.890(0.381-2.078)$ & 0.788 & & \\
\hline HAS2 cancer $(+)$ & $0.886(0.425-1.850)$ & 0.748 & & \\
\hline
\end{tabular}

HPV, human papilloma virus; HR, hazard ratio; CI, confidence interval; HA, hyaluronic acid; HYAL1, hyaluronidase 1; HAS2, HA synthases 2 . 
Oral cavity cancer

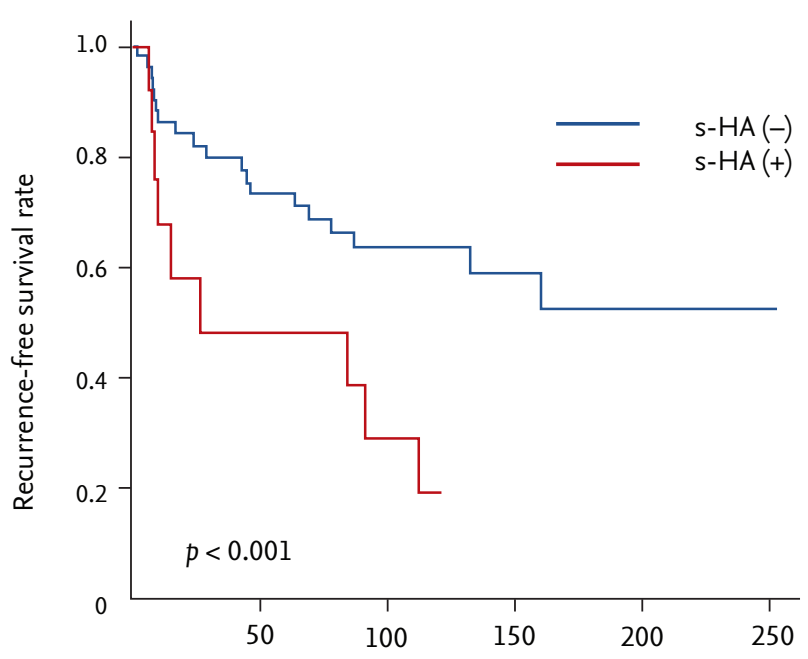

A

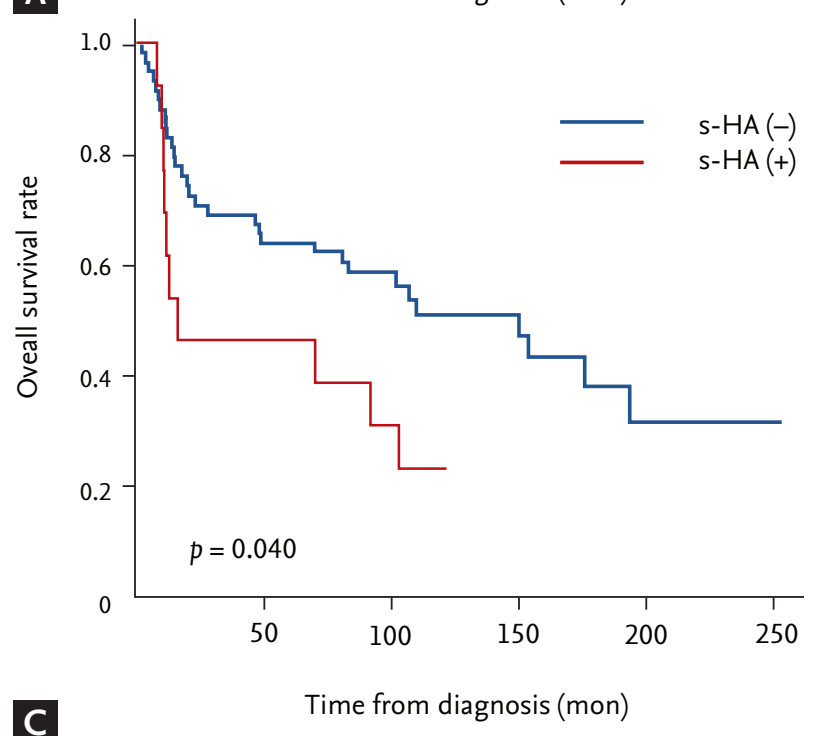

Oropharyngeal cancer

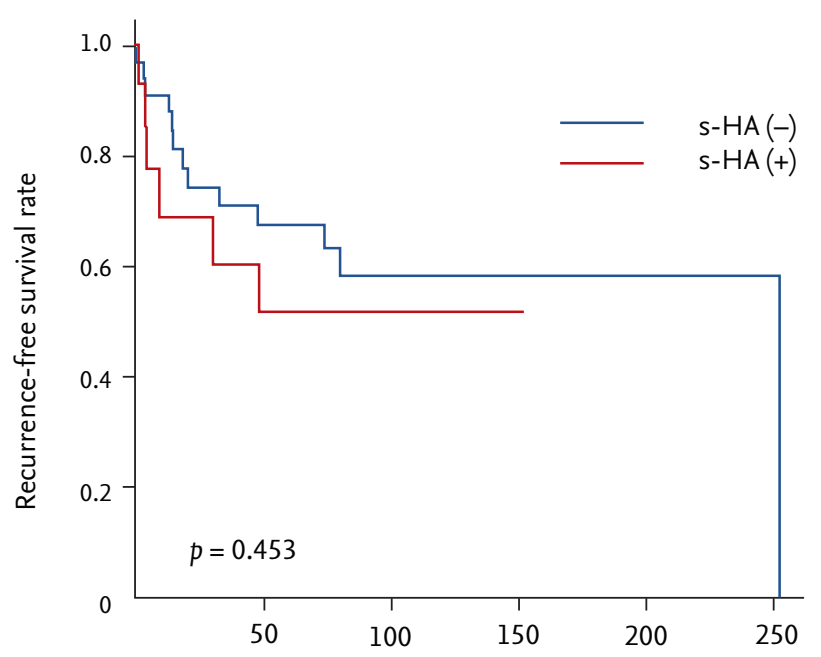

B

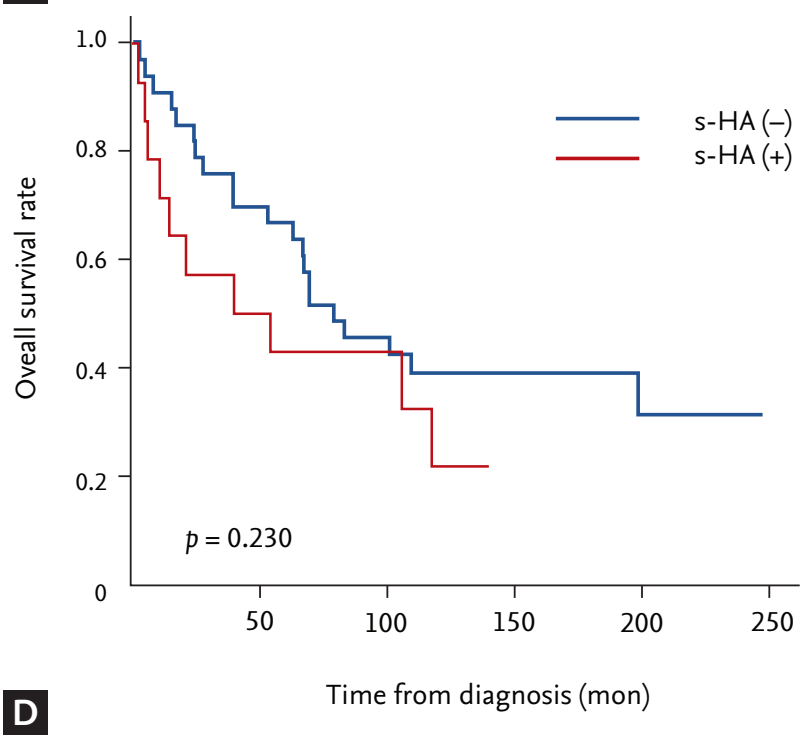

Figure 2. Kaplan-Meier curves for recurrence-free survival (A, B) and overall survival (C, D) according to stromal hyaluronic acid (s-HA) expression in human papilloma virus-negative oral cavity cancer (A, C) and oropharyngeal (B, D) patients.

actions with HA receptors.

Previous studies found high levels of HA around tumor cells or within the tumor stroma. The levels of HA in tumor cells and/or stroma were correlated with overall tumor aggressiveness and increased cell migration and proliferation in various types of cancer [14]. Auvinen et al. [10] reported that both HA associated with tumor cells and stromal HA were independent prognostic factors for OS in patients with breast cancer. Lokeshwar et al. [15] reported that stromal-epithelial HA overexpres- sion may be linked with cancer progression. In addition, in gastric and colorectal cancers, high levels of HA in tumor cells was shown to be a poor prognostic factor associated with reduced survival [8,9]. There have been few studies evaluating HA in head and neck cancer. Kosunen et al. [16] reported that homogeneous expression of HA in tumor epithelium was associated with better OS and disease-free survival compared with irregular HA expression. Hirvikoski et al. [17] reported the prognostic role of tumoral HA in laryngeal squamous cell carcino- 
Oral cavity cancer
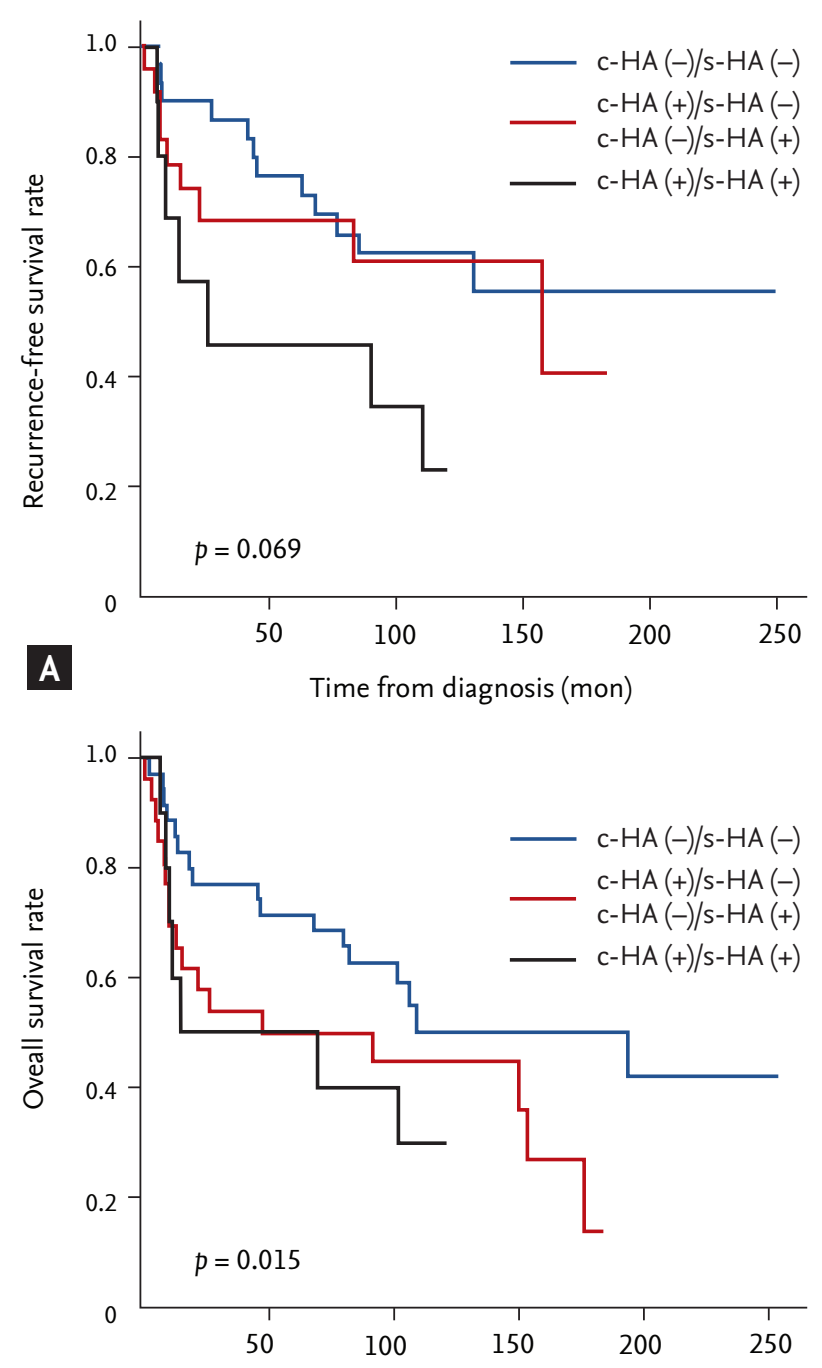

C
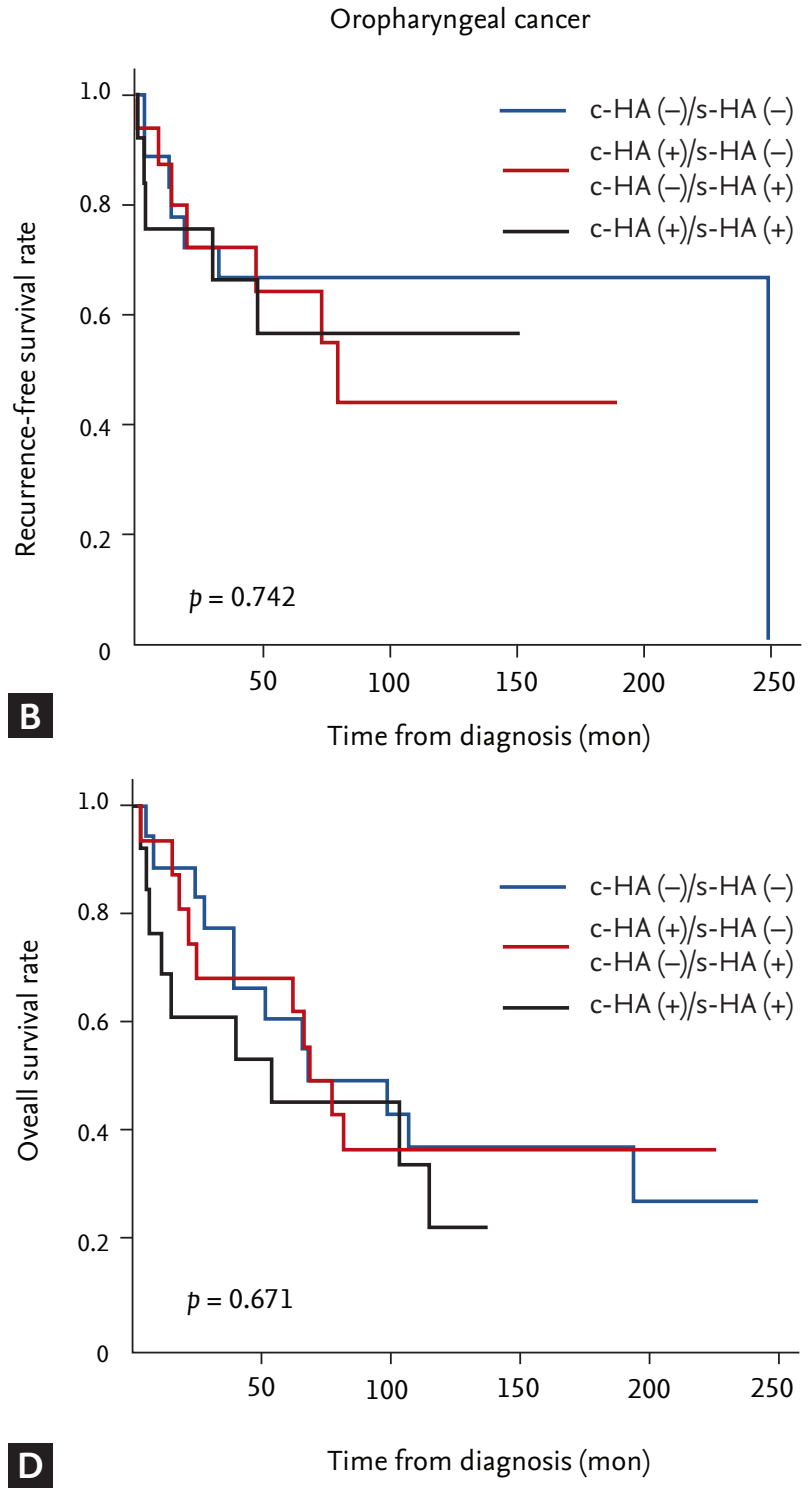

Figure 3. Kaplan-Meier curves for recurrence-free survival (A, B) and overall survival (C, D) according to the combined expression of tumoral hyaluronic acid (c-HA) and stromal hyaluronic acid (s-HA) in human papilloma virus-negative oral cavity cancer $(\mathrm{A}, \mathrm{C})$ and oropharyngeal cancer $(\mathrm{B}, \mathrm{D})$ patients.

ma using intensity scores for homogeneous or irregular staining. They showed that the irregular staining of HA in tumor cells of laryngeal squamous cell carcinoma trended toward poor disease-free survival, suggesting that an altered HA metabolism is associated with an aggressive growth pattern [17]. In the present study, we first identified an association between high stromal HA expression and poor prognosis in patients with HPV-negative oral cavity cancer. Interestingly, tumor stromal HA expression was only observed when tumor cells exhibited tumoral HA expression. Lokeshwar et al. [15] demonstrated that HA is localised almost exclusively in the stroma and its concentration increased considerably in tumor-associated stroma in prostate cancer, and both stromal fibroblasts and tumor epithelial cells from prostate cancer tissues showed elevated HA secretions. Based on these findings and the intimate crosstalk between tumors and the tumor microenvironment, HA expression may result from the interaction between tumors and tumor stroma. In this study, we observed 


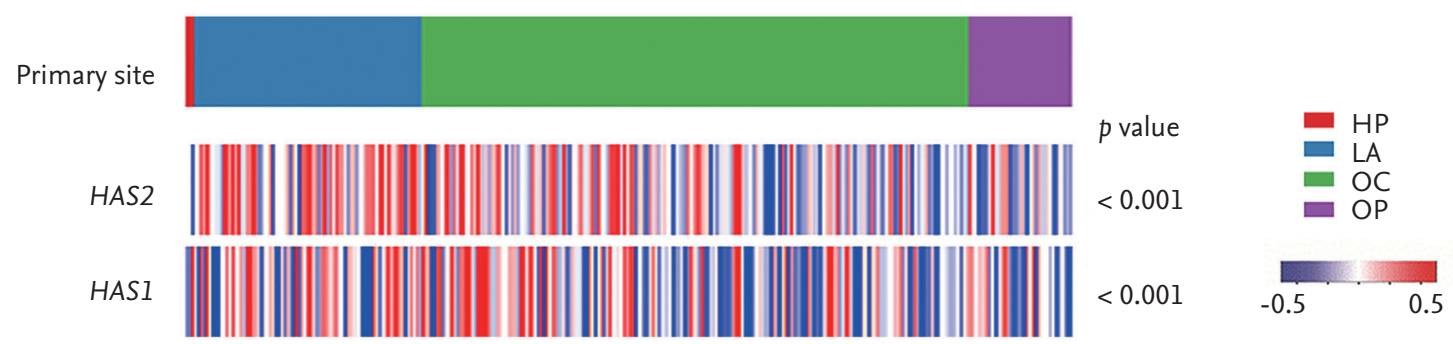

A

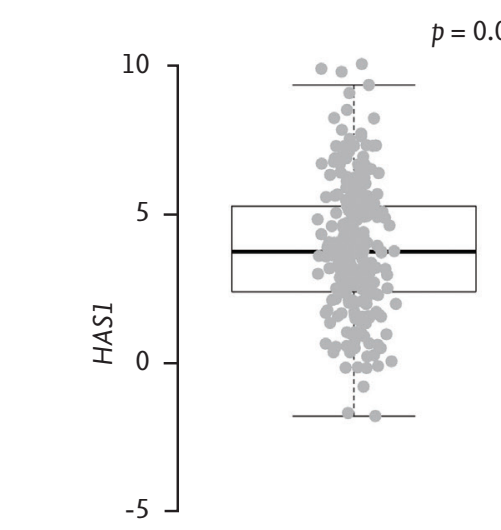

B

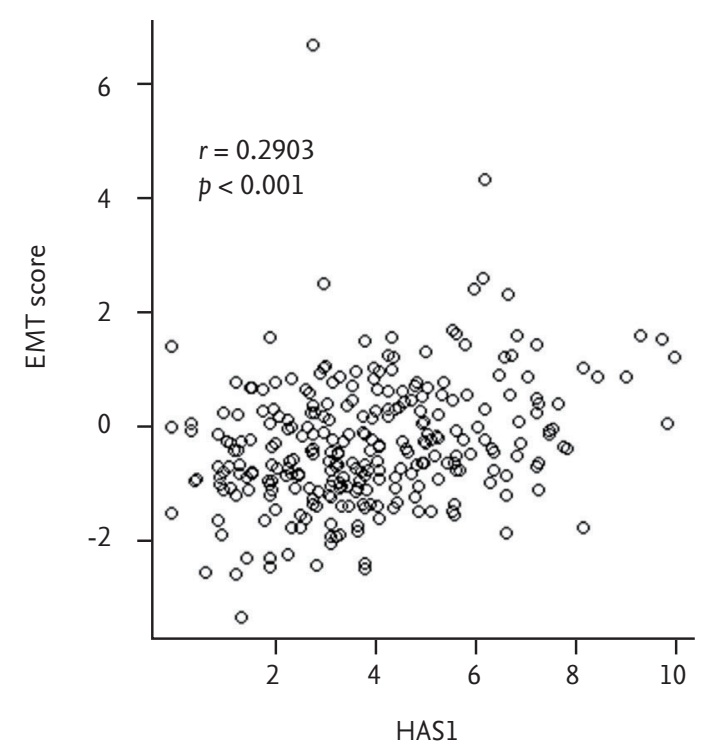

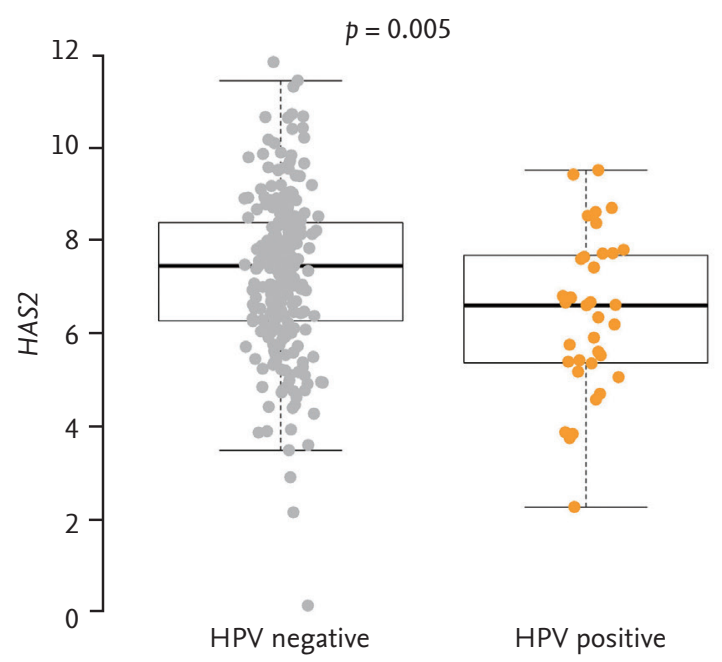

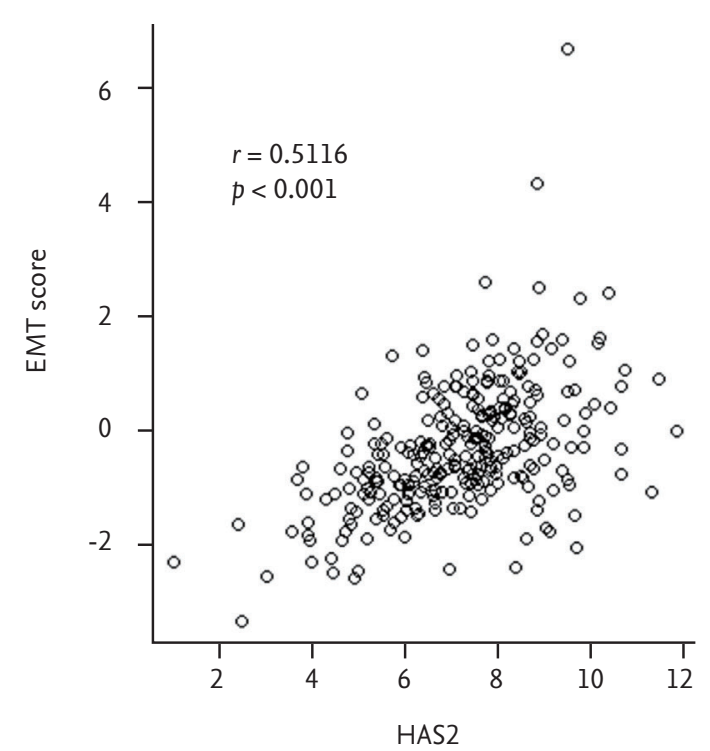

Figure 4. Hyaluronic acid synthase $2\left(H_{A S 2}\right)$ and HAS 1 gene expression in The Cancer Genome Atlas (TCGA) head and neck squamous cell carcinoma (HNSCC) database $(n=279)$. The levels of HAS2 and HAS 1 gene expression according to (A) the primary site, (B) human papilloma virus (HPV) status, and (C) epithelial-mesenchymal transition (EMT) score. HP, hypopharynx; LA, larynx; OC, oral cavity; OP, oropharynx. 
that tumoral and stromal HA expression were associated with poor prognosis when they were simultaneously expressed in cancer tissues.

HYAL1 is the major tumor-derived HAase that is almost exclusively expressed in tumor cells. The expression and function of HYALı has been widely investigated in many solid cancer types. Kramer et al. [18] detected the combined HAS2-HYALı biomarker in bladder cancer and found it significantly predicted recurrence. In another study, upregulation of HYALı in breast cancer promoted tumor cell proliferation, migration, invasion, and angiogenesis $[19,20]$. In breast cancer, HAS2 has shown a critical role in the development of a prometastatic microenvironment, based on highly metastatic cancer stem-like cells and aggressive phenotypes, by increasing transforming growth factor-beta-induced EMT $[21,22]$. However, there are few data on the roles of HAS2 and HYAL1 in HNSCC. Zhang et al. [23] reported that HAS2 was one of the key regulators responsible for cancer-associated fibroblast-mediated OSCC progression. Unlike previous studies of other cancer types, we did not find a prognostic role of HYAL1 and HAS2 in HNSCC, regardless of HPV status. Further studies are needed to clarify this in a homogeneous group. Analysis of TCGA HNSCC data showed that HA-related genes (HAS2 and $H A S 1)$ were significantly correlated with EMT, suggesting that HA-related genes may be involved in the development of the aggressive phenotype of HNSCC through EMT.

HNSCC has two representative pathogenic routes: (1) chemical exposure, including tobacco and alcohol, and (2) high-risk HPV-induced carcinogenesis. HPV-positive HNSCC is a biologically distinct entity with a unique molecular pathogenesis $[24,25]$. With advances in comprehensive molecular analysis methods, Chung et al. [26] reported the molecular classification of HNSCC using gene expression patterns (classical, basal, mesenchymal, and atypical groups). Based on this molecular classification and pathogenesis, HPV-negative HNSCC is expected to have a stronger mesenchymal cell signature than HPV-positive HNSCC [25,26]. This may be one reason for the stronger prognostic impact of stromal HA expression in HPV-negative tumors. Indeed, in an analysis of TCGA HNSCC data, oral cavity cancers and HPV-negative tumors, mostly composed of the mesenchymal subtype, were closely related in the upregulated

\section{HAS2 or HAS1 tumors.}

Recently, methods targeting HA, including the inhibition of HA synthesis, blocking HA-receptor signalling and depletion of stromal HA, have been recognised as promising cancer treatment strategies. HA inhibition normalises interstitial pressure and re-expands the microvasculature, consequently overcoming the stromal barrier [27]. These changes enhance the sensitivity and efficiency of chemotherapy. Recent studies have shown that pegvorhyaluronidase alfa (PEGPH2O), a HA-targeting enzymatic agent, degrades HA and increases drug delivery. This agent improved the effects of chemotherapy with gemcitabine in animal models [28]. In randomised phase 2 clinical trial comparing nab-paclitaxel/ gemcitabine with PEGPH2o versus without PEGPH2O in metastatic pancreatic cancer, significant improvement of progression-free survival was observed in patients with HA-high tumors who received PEGPH2O [29,30].

In conclusion, stromal HA expression was an independent factor for poor prognosis in terms of RFS in HPV-negative oral cavity cancer. Further preclinical and clinical studies are needed to more clearly elucidate the potential therapeutic role of HA-targeting agents, such as PEGPH2O in HPV-negative oral cavity cancer.

\section{KEY MESSAGE}

1. Hyaluronic acid (HA) and HA family molecules, such as HA synthase, HA receptors and hyaluronidases, are associated with cell proliferation, invasion, and migration in various cancers.

2. We found that high stromal HA expression was an independent factor associated with a poor prognosis in terms of recurrence-free survival (RFS) in human papilloma virus (HPV)-negative oral cavity cancers.

3. The expression of HA in both tumors and stroma was significantly correlated with shorter RFS than other combinations in patients with HPV-negative oral cavity cancer. Therefore, the prognostic impact of stromal HA expression may be much stronger in HPV-negative tumors than in HPV-positive tumors. 


\section{Conflict of interest}

No potential conflict of interest relevant to this article was reported.

\section{Acknowledgments}

This study was supported by the Basic Science Research Pro-gram through the National Research Foundation of Korea (NRF) funded by the Ministry of Science, ICT \& Future Planning (NRF-2015R1C1A1A01054591) (Yoon Ho Ko) and by The Catholic University of Korea Uijeongbu St. Mary's Hospital Clinical Research Laboratory Foundation made in the program year of 2013 (Yoon Ho Ko).

\section{REFERENCES}

1. Warnakulasuriya S. Global epidemiology of oral and oropharyngeal cancer. Oral Oncol 2009;45:309-316.

2. Markovic A, Chung CH. Current role of EGF receptor monoclonal antibodies and tyrosine kinase inhibitors in the management of head and neck squamous cell carcinoma. Expert Rev Anticancer Ther 2012;12:1149-1159.

3. Carinci F, Stabellini G, Calvitti M, et al. CD44 as prognostic factor in oral and oropharyngeal squamous cell carcinoma. J Craniofac Surg 2002;13:85-89.

4. McDonald JA, Camenisch TD. Hyaluronan: genetic insights into the complex biology of a simple polysaccharide. Glycoconj J 2002;19:331-339.

5. Itano N, Kimata K. Mammalian hyaluronan synthases. IUBMB Life 2002;54:195-199.

6. Stern R. Hyaluronan catabolism: a new metabolic pathway. Eur J Cell Biol 2004;83:317-325.

7. Wang C, Tammi M, Guo H, Tammi R. Hyaluronan distribution in the normal epithelium of esophagus, stomach, and colon and their cancers. Am J Pathol 1996;148:18611869.

8. Ropponen K, Tammi M, Parkkinen J, et al. Tumor cell-associated hyaluronan as an unfavorable prognostic factor in colorectal cancer. Cancer Res 1998;58:342-347.

9. Setala LP, Tammi MI, Tammi RH, et al. Hyaluronan expression in gastric cancer cells is associated with local and nodal spread and reduced survival rate. $\mathrm{Br} \mathrm{J}$ Cancer 1999;79:1133-1138.

10. Auvinen P, Tammi R, Parkkinen J, et al. Hyaluronan in peritumoral stroma and malignant cells associates with breast cancer spreading and predicts survival. Am J
Pathol 2000;156:529-536.

11. Byers LA, Diao L, Wang J, et al. An epithelial-mesenchymal transition gene signature predicts resistance to EGFR and $\mathrm{PI}_{3} \mathrm{~K}$ inhibitors and identifies Axl as a therapeutic target for overcoming EGFR inhibitor resistance. Clin Cancer Res 2013;19:279-290.

12. Lassen P, Primdahl H, Johansen J, et al. Impact of HPV-associated p16-expression on radiotherapy outcome in advanced oropharynx and non-oropharynx cancer. Radiother Oncol 2014;113:310-316.

13. Li L, Asteriou T, Bernert B, Heldin CH, Heldin P. Growth factor regulation of hyaluronan synthesis and degradation in human dermal fibroblasts: importance of hyaluronan for the mitogenic response of PDGF-BB. Biochem J 2007;404:327-336.

14. Toole BP. Hyaluronan: from extracellular glue to pericellular cue. Nat Rev Cancer 2004;4:528-539.

15. Lokeshwar VB, Rubinowicz D, Schroeder GL, et al. Stromal and epithelial expression of tumor markers hyaluronic acid and HYALı hyaluronidase in prostate cancer. J Biol Chem 2001;276:11922-11932.

16. Kosunen A, Ropponen K, Kellokoski J, et al. Reduced expression of hyaluronan is a strong indicator of poor survival in oral squamous cell carcinoma. Oral Oncol 2004;40:257-263.

17. Hirvikoski P, Tammi R, Kumpulainen E, et al. Irregular expression of hyaluronan and its $\mathrm{CD}_{44}$ receptor is associated with metastatic phenotype in laryngeal squamous cell carcinoma. Virchows Arch 1999;434:37-44.

18. Kramer MW, Escudero DO, Lokeshwar SD, et al. Association of hyaluronic acid family members (HAS1, HAS2, and HYAL-1) with bladder cancer diagnosis and prognosis. Cancer 2011;117:1197-1209.

19. Tan JX, Wang XY, Su XL, et al. Upregulation of HYALı expression in breast cancer promoted tumor cell proliferation, migration, invasion and angiogenesis. PLoS One 2011;6:e22836.

20. McAtee CO, Barycki JJ, Simpson MA. Emerging roles for hyaluronidase in cancer metastasis and therapy. Adv Cancer Res 2014;123:1-34.

21. Okuda H, Kobayashi A, Xia B, et al. Hyaluronan synthase HAS2 promotes tumor progression in bone by stimulating the interaction of breast cancer stem-like cells with macrophages and stromal cells. Cancer Res 2012;72:537547.

22. Lien HC, Lee YH, Jeng YM, et al. Differential expression 
of hyaluronan synthase 2 in breast carcinoma and its biological significance. Histopathology 2014;65:328-339.

23. Zhang Z, Tao D, Zhang P, et al. Hyaluronan synthase 2 expressed by cancer-associated fibroblasts promotes oral cancer invasion. J Exp Clin Cancer Res 2016;35:181.

24. Kang H, Kiess A, Chung CH. Emerging biomarkers in head and neck cancer in the era of genomics. Nat Rev Clin Oncol 2015;12:11-26.

25. Cancer Genome Atlas Network. Comprehensive genomic characterization of head and neck squamous cell carcinomas. Nature 2015;517:576-582.

26. Chung CH, Parker JS, Karaca G, et al. Molecular classification of head and neck squamous cell carcinomas using patterns of gene expression. Cancer Cell 2004;5:489-500.

27. Provenzano PP, Cuevas C, Chang AE, Goel VK, Von Hoff
DD, Hingorani SR. Enzymatic targeting of the stroma ablates physical barriers to treatment of pancreatic ductal adenocarcinoma. Cancer Cell 2012;21:418-429.

28. Thompson CB, Shepard HM, O'Connor PM, et al. Enzymatic depletion of tumor hyaluronan induces antitumor responses in preclinical animal models. Mol Cancer Ther 2010;9:3052-3064.

29. Sato N, Cheng XB, Kohi S, Koga A, Hirata K. Targeting hyaluronan for the treatment of pancreatic ductal adenocarcinoma. Acta Pharm Sin B 2016;6:101-105.

30. Hingorani SR, Zheng L, Bullock AJ, et al. HALO 202: randomized phase II study of PEGPH2o plus nab-paclitaxel/gemcitabine versus nab-paclitaxel/gemcitabine in patients with untreated, metastatic pancreatic ductal adenocarcinoma. J Clin Oncol 2018;36:359-366. 